\title{
Nível de evidência e patrocínio industrial associado a desfechos favoráveis nas publicações sobre terapia com plasma rico em plaquetas nas doenças osteomusculares*
}

\section{Level of Evidence and Industry Sponsorship Associated with Favorable Outcomes in Publications on Platelet-Rich Plasma Therapy in Musculoskeletal Disorders}

\author{
Pietro Felice Tomazini Nesello ${ }^{10}$ Allan Cassio Baroni ${ }^{1}$ Luciano da Silva Selistre ${ }^{2}$ \\ ${ }^{1}$ Instituto de Medicina do Esporte e Ciências Aplicadas ao Movimento \\ Humano, Universidade de Caxias do Sul, Caxias do Sul, RS, Brasil \\ 2 Departamento de Bioestatística, Hospital Geral, Universidade de \\ Caxias do Sul, Caxias do Sul, RS, Brasil \\ Endereço para correspondência Pietro Felice Tomazini Nesello, MD, \\ Instituto de Medicina do Esporte e Ciências Aplicadas ao Movimento \\ Humano, Universidade de Caxias do Sul, Rua Francisco Getúlio Vargas \\ 1.130, Caxias do Sul, RS, 95070-560, Brasil \\ (e-mail: pietrofelicenesello@gmail.com).
}

Rev Bras Ortop 2020;55(3):263-268.

\begin{abstract}
Resumo
O plasma rico em plaquetas é derivado da centrifugação do sangue total. Há um interesse crescente, na medicina esportiva e na comunidade atlética, no fornecimento de fatores de crescimento endógeno diretamente ao sítio da lesão, usando componentes sanguíneos autólogos, como o plasma rico em plaquetas. O objetivo deste estudo é avaliar a associação entre financiamento de pesquisa, conflito de interesses, nível de evidência e afiliação dos autores com a interpretação dos resultados em publicações sobre terapia com plasma rico em plaquetas nas doenças osteomusculares. Foi realizada uma revisão da literatura atual. O desfecho foi classificado como favorável ou desfavorável. A declaração de conflito de interesses e o tipo de financiamento foram extraídos de cada artigo. O financiamento foi qualificado em patrocínio industrial; não patrocinado pela indústria; ou não identificável. O nível de evidência foi

Palavras-chave

- plasma rico em plaquetas

- doenças musculoesqueléticas

- indústria

- conflito de interesses

- ética categorizado de I a IV. Foram obtidos os resultados positivos mais altos com 134 estudos financiados pelo setor industrial, em comparação com estudos não financiados pela indústria (razão de probabilidades [RP]: 0,26; intervalo de confiança de 95\% [95\% IC]: 0,08-0,85; $p<0,05)$. Em comparação com o nível de evidência I, os níveis II e IV aumentam a probabilidade de resultado positivo em 12,42 vezes $(p<0,01)$ e 10,97 vezes $(p<0,01)$, respectivamente. Demonstrou-se que, proporcionalmente, estudos patrocinados pela indústria têm maior probabilidade de apresentar resultados positivos, bem como artigos com menor qualidade de evidência.
\end{abstract}

\footnotetext{
Trabalho realizado no Instituto de Medicina do Esporte e Ciências Aplicadas ao Movimento Humano, Universidade de Caxias do Sul, RS, Brasil.
}

recebido

24 de Abril de 2018

aceito

16 de Outubro de 2018
DOI https://doi.org/

10.1055/s-0039-1700834. ISSN 0102-3616.
Copyright $\odot 2020$ by Sociedade Brasileira License terms de Ortopedia e Traumatologia. Published by Thieme Revinter Publicações Ltda, Rio de Janeiro, Brazil 


\begin{abstract}
Platelet-rich plasma is derived from centrifuging whole blood. There is increasing interest in the sports medicine and athlete community about providing endogenous growth factors directly to the injury site, using autologous blood products such as platelet-rich plasma. The aim of the present study is to evaluate the association between research financing, conflict of interests, level of evidence and author affiliation with the interpretation of results in articles published on platelet-rich plasma therapy in musculoskeletal ailments. A review of the current literature was performed. The outcome was classified as favorable or unfavorable. The declaration of conflict of interests and the type of funding was extracted from each article. The financing was classified as industry-sponsored; not industry-sponsored; or unidentifiable. The level of

\section{Keywords}

- platelet-rich plasma

- musculoskeletal diseases

- industry

- conflict of interests

- ethics evidence was categorized from I to IV. Higher positive outcomes were observed in 134 studies with industry sponsorship compared with not industry-sponsored studies (odds ratio [OR]: 0.26; $95 \%$ confidence interval [95\%Cl]: 0.08-0.85; $p<0.05$ ). Compared with level of evidence I, levels II and IV increase the probability of positive outcomes by 12.42 times $(p<0.01)$ and 10.97 times $(p<0.01)$ respectively. Proportionally, industrysponsored studies are more likely to present positive results, as well as articles with a lower quality of evidence.
\end{abstract}

\section{Introdução}

O plasma rico em plaquetas (PRP) é derivado da centrifugação do sangue do paciente, resultando em uma fração rica em plaquetas maior do que a concentração sérica. ${ }^{1} \mathrm{~A}$ terapia com esta preparação consiste na ativação de fatores de crescimento que migram para o sítio doente, ou seja, a região onde é necessária uma evolução da regeneração tecidual e da angiogênese. Desta forma, o efeito biológico dessa preparação do sangue toma forma, facilitando o ambiente biocelular e, teoricamente, acelerando o processo de cicatrização. ${ }^{2}$

Recentemente, o uso do PRP foi observado no tratamento de algumas lesões osteomusculares. ${ }^{3,4}$ No entanto, muitos desses estudos apresentam um tamanho de amostra pequeno, com alto risco de viés. ${ }^{5-7}$ Sabe-se que 0 apoio financeiro industrial está associado a achados favoráveis em publicações de diversos estudos referentes à cirurgia ortopédica. ${ }^{8,9}$

O objetivo deste artigo é descrever e investigar a associação entre financiamento da pesquisa, conflito de interesses, nível de evidência e afiliação dos autores com a interpretação dos resultados em publicações sobre a terapia com PRP em doenças osteomusculares (DOMs).

\section{Métodos}

\section{Estratégia de Pesquisa}

Foram incluídos os artigos sobre terapia com PRP relacionados às DOMs e publicados em periódicos. Em seguida, foi realizada uma revisão das bases de dados PubMed e Scielo. A pesquisa de artigos publicados nos últimos 10 anos foi realizada com palavras-chave no título e finalizada em 12 de agosto de 2016. Foram pesquisadas as seguintes palavras-chave: Platelet rich plasma[Title] AND (hamstring[Title] OR achilles[Title] OR tunnel[Title] OR patellar[Title] OR plantar[Title] OR talar[Title] OR talus[Title] OR calcaneal[Title] OR calcaneus[Title] OR cruciate[Title] OR ulnar[Title] OR radial[Title] OR tibial[Title] OR knee
[Title] OR shoulder[Title] OR elbow[Title] OR ankle[Title] OR hip [Title] OR rotator cuff[Title] OR handle[Title] OR low back[Title] OR spinal[Title] OR cervical[Title] OR arm[Title] OR forearm [Title] OR gluteal[Title] OR gluteus[Title] OR calf[Title] OR leg [Title] OR gastrocnemius[Title] OR quadriceps[Title] OR abductor [Title] OR adductor[Title] OR abdominal[Title] OR biceps[Title]OR triceps[Title] OR pectoral[Title] OR joint[Title] OR articular[Title] OR chondral[Title] OR tendon[Title] OR tendinous[Title] OR soft tissue[Title] OR muscle[Title] OR muscles[Title] OR muscular [Title] OR musculoskeletal[Title] OR bone[Title] OR bones[Title] OR skeletal[Title] OR cartilage[Title] OR cartilaginous[Title] OR ligament[Title] OR ligaments[Title] OR osteochondral[Title] OR damage[Title] OR damages[Title] OR harm[Title] OR harms[Title] OR contusion[Title] OR contusions[Title] OR sprain[Title] OR sprains[Title] OR twist[Title] OR twists[Title] OR torsion[Title] OR torsions[Title] OR fractures[Title] OR rupture[Title] OR ruptures[Title] OR dislocation[Title] OR dislocations[Title] OR luxation[Title] OR luxations[Title] OR strain[Title] OR strains[Title] OR tendinitis[Title] OR tendinopathy[Title] OR tendinopathies [Title] OR tendinosis[Title] OR fasciitis[Title] OR arthritis[Title] OR osteoarthritis[Title] OR arthrosis[Title] OR osteoarthrosis [Title] OR osteoporosis[Title] OR osteomyelitis[Title] OR bursitis [Title] OR lesion[Title] OR lesions[Title] OR synovitis[Title] OR trauma[Title] OR traumatic[Title] OR injury[Title] OR injuries [Title] OR epicondylitis[Title] OR sport[Title] OR sports[Title] OR athletes[Title] OR degenerative[Title])AND("2008/09/23"[PDat]: “2016/08/12"[PDat] AND “humans"[MeSH Terms] AND (Portuguese[lang] OR English[lang]) AND “adult"[MeSH Terms]).

Os critérios de inclusão foram: trabalhos publicados sobre terapia com PRP em DOMs, de pesquisas em humanos $>18$ anos, escritos em inglês ou português, e com texto completo disponível. Os critérios de exclusão foram: trabalhos que tratavam de tema diferente, estudos experimentais e de protocolo, e com qualidade de evidência nível $\mathrm{V}$ na escala de Oxford. ${ }^{10}$ 


\section{Coleta de Dados}

Dois autores revisaram de forma independente cada artigo, e coletaram os dados: qualidade dos resultados, nível de evidência, autoria, financiamento e conflito de interesses.

De acordo com as hipóteses dos autores de cada artigo, as conclusões e discussões foram revisadas para avaliar a interpretação dos resultados. Expressões-chave como "mais eficaz", "superior", "é melhor", “mais eficiente”, “é seguro”, "é recomendado", "deve ser usado" qualificaram o resultado como favorável. Em seguida, as expressões: "é o mesmo", "não exibiu diferença”, e "não há evidências para apoiar o uso" denotaram um resultado desfavorável. Cada artigo foi avaliado de acordo com as diretrizes do Oxford Center for Clinical Evidence. ${ }^{10} \mathrm{~A}$ classificação do nível de evidência variou de I a V, sendo o nível I a mais alta qualidade, e o V, a mais baixa. A autoria, por sua vez, foi considerada acadêmica caso todos os autores apresentassem afiliação acadêmica: faculdade de medicina, universidade ou hospital. Se um ou mais autores divulgaram afiliação laboratorial, industrial ou a qualquer outra empresa, esse tipo de autoria foi considerada industrial. O financiamento do estudo foi categorizado da seguinte forma: patrocinado pela indústria, não patrocinado pela indústria, ou não identificável. Se algum dos autores era vinculado a uma empresa farmacêutica, caso o apoio financeiro tenha sido declarado ou tenha havido gratidão expressa a qualquer setor, o estudo foi considerado patrocinado pela indústria. No caso de apoio financeiro de órgãos públicos, governamentais, acadêmicos e de pesquisa, o financiamento foi considerado não patrocinado pela indústria. Quando não foi possível classificá-lo, o apoio financeiro foi considerado como não identificável. A declaração de conflito de interesses foi classificada como presente ou ausente. No caso de ela não existir, o conflito de interesses não foi classificado.

Os casos em que os autores deste artigo não chegaram a um acordo foram revisados e discutidos até que o acordo fosse alcançado.

\section{Análise Estatística}

O nível de concordância entre os observadores foi medido por meio da estatística capa (к), e os valores foram descritos de acordo com os critérios de Fleiss. ${ }^{11}$ Foi utilizada uma estatística descritiva com frequências e porcentagens. O teste do Quiquadrado foi utilizado para avaliar a relação entre a qualidade do resultado e as demais variáveis. Uma regressão lógica binária posterior foi planejada e usada para diminuir o viés de confusão, medindo, assim, a razão de probabilidades [RP] ajustada (exponenciação do coeficiente $\mathrm{B}$ [Exp B]). As variáveis com valor de $p<0,20$ no teste do Qui-quadrado foram selecionadas para regressão logística binária. Todas as análises estatísticas foram realizadas com o programa Statistical Package for the Social Sciences (SPSS, IBM Corp., Armonk, NY, EUA), versão 22 . O valor de $p$ definido como $<0,05$ de ambos os lados foi considerado significativo com um intervalo de confiança de 95\% (IC95\%).

\section{Resultados}

Nossa pesquisa eletrônica identificou 204 estudos publicados desde 16 de agosto de 2006. Após excluir 70 (34,3\%) estudos, 134
Tabela 1 Fluxograma da estratégia de busca para identificar estudos sobre o uso da terapia rica em plaquetas em doenças do sistema musculoesquelético

\begin{tabular}{|l|}
\hline 1. Identificação \\
\hline 2. Seleção \\
\hline 3. Elegibilidade \\
\hline 4. Incluídos \\
\hline 5. Artigos encontrados na busca eletrônica $(\mathrm{n}=204)$ \\
\hline 6. Artigos selecionados para revisão $(\mathrm{n}=188)$ \\
\hline 7. Artigos avaliados para elegibilidade $(\mathrm{n}=134)$ \\
\hline 8. Estudos incluídos ( $\mathrm{n}=134)$ \\
\hline 9. Excluídos (16): \\
\hline • Idioma espanhol (3) e tcheco (1); \\
\hline • Texto completo não disponível $(12) ;$ \\
\hline 10. Excluídos (54): \\
\hline • Não relacionados ao tema da pesquisa $(26) ;$ \\
\hline • Estudos de protocolo (2); \\
\hline • Estudos experimentais/laboratoriais (20); \\
\hline • Nível V de evidência (6); \\
\hline
\end{tabular}

(65,7\%) publicações foram incluídas para análise (-Tabela $\mathbf{1}$ ). A frequência de desfechos favoráveis foi de 96 (71,6\%), diferindo significativamente do desfecho negativo $(p<0,001)$. 0 valor de $\mathrm{K}$ interobservador para o desfecho foi de $0,89(p<0,001)$, apresentando um índice de excelência.

A indústria foi identificada como patrocinadora do estudo em 26,1\% dos casos ( - Tabela 2); no entanto, em quase metade dos casos, não foi possível classificar o financiador. Havia conflito de interesses em $15,7 \%$ do total, e a maior proporção de artigos tinha nível IV na escala de Oxford. Salienta-se que em todas as variáveis descritas, as frequências esperadas foram diferentes ( $p<0,001)$. Os índices $\mathrm{k}$ estavam todos na categoria marcada como "excelente" ( $p<0,001)$.

A relação entre as variáveis investigadas denota que os estudos patrocinados pela indústria apresentaram maior frequência de resultados positivos em relação aos não patrocinados pela indústria. Por outro lado, em relação aos níveis de evidência, observa-se significativamente $(p<0,001)$ que quanto mais desqualificado um estudo, maior a frequência de resultados positivos (- Tabela $\mathbf{3}$ ).

Na análise multivariada, as variáveis participantes do modelo foram: financiamento e nível de evidência. A estatística geral para o Qui-quadrado dos resíduos foi de 27,44 ( $p<0,001)$. A partir da análise do modelo, foi detectada uma relação significativa entre o financiamento industrial e o financiamento não patrocinado pela indústria. A RP ajustada para um resultado positivo diminuiu em $\sim 74 \%$ no estudo não industrial em relação ao estudo patrocinado pela indústria (RP: 0,26; IC95\%: $0,08-0,85 ; p<0,05)$. Por outro lado, quanto ao nível de evidência, observou-se que, comparado ao nível de evidência I, os níveis II e IV aumentaram (RP: 12,42; IC95\%: 3,79-40,67; $p<0,001$; e RP: 10,97 ; IC95\%: 2,33-51,51; $p<0,01)$, respectivamente, para um resultado positivo ( - Tabela 4 ). 
Tabela 2 Frequência esperada e nível de concordância entre observadores

\begin{tabular}{|c|c|c|c|c|c|c|}
\hline & & Frequência (\%) & Qui-quadrado* & Valor de $p$ & Valor de capa (к) & Valor de $p$ \\
\hline \multirow[t]{2}{*}{ Resultado } & Favorável & $96(71,6)$ & 25,10 & $<0,001$ & 0,89 & $<0,001$ \\
\hline & Desfavorável & $38(27,9)$ & & & & \\
\hline \multirow[t]{3}{*}{ Financiamento } & Industrial & $35(26,1)$ & 25,80 & $<0,001$ & 0,85 & $<0,001$ \\
\hline & Não industrial & $27(20,1)$ & & & & \\
\hline & Não classificado & $72(53,7)$ & & & & \\
\hline \multirow[t]{2}{*}{ Autoria } & Acadêmica & $46(34,3)$ & 13,16 & $<0,001$ & 0,83 & $<0,001$ \\
\hline & Industrial & $88(65,7)$ & & & & \\
\hline \multirow[t]{3}{*}{ Conflito de interesses } & Sim & $21(15,7)$ & 39,50 & $<0,001$ & 0,87 & $<0,001$ \\
\hline & Não & $78(58,2)$ & & & & \\
\hline & Não classificado & $35(26,1)$ & & & & \\
\hline \multirow[t]{4}{*}{ Nível de evidência } & 1 & $27(20,1)$ & 38,32 & $<0,001$ & 0,80 & $<0,001$ \\
\hline & II & $39(29,1)$ & & & & \\
\hline & III & $10(7,5)$ & & & & \\
\hline & IV & $58(43,3)$ & & & & \\
\hline
\end{tabular}

Nota: *Análise do qui-quadrado para uma amostra da frequência equivalente esperada.

Tabela 3 Associação entre a qualidade do resultado e as variáveis estudadas

\begin{tabular}{|c|c|c|c|c|}
\hline & & \multicolumn{2}{|l|}{ Resultado } & \multirow[t]{2}{*}{ Valor de $p$} \\
\hline & & Favorável $(n=96)$ & Desfavorável $(n=38)$ & \\
\hline \multirow[t]{3}{*}{ Financiamento } & Patrocinado pela indústria $(n=35)$ & $29(30,2 \%)$ & $6(15,8 \%)$ & \multirow[t]{3}{*}{0,159} \\
\hline & $\begin{array}{l}\text { Não patrocinado pela indústria } \\
(n=27)\end{array}$ & $20(20,8 \%)$ & $7(18,4 \%)$ & \\
\hline & Não identificável $(n=72)$ & 47 (49\%) & $25(65,8 \%)$ & \\
\hline \multirow[t]{2}{*}{ Autoria } & $\begin{array}{l}\text { Acadêmica } \\
(n=46)\end{array}$ & $31(32,3 \%)$ & $15(39,5 \%)$ & 0,430 \\
\hline & Industrial $(n=88)$ & $65(67,7 \%)$ & $23(60,5 \%)$ & \\
\hline \multirow[t]{3}{*}{ Conflito de interesses } & $\operatorname{Sim}(n=21)$ & $16(16,7 \%)$ & $5(13,2 \%)$ & \multirow[t]{3}{*}{0,638} \\
\hline & Não $(n=78)$ & $57(59,4 \%)$ & $21(55,3 \%)$ & \\
\hline & $\begin{array}{l}\text { Não classificado } \\
(n=35)\end{array}$ & $23(24 \%)$ & $12(31,6 \%)$ & \\
\hline \multirow[t]{4}{*}{ Nível de evidência } & $\mathrm{I}(n=27)$ & $12(12,5 \%)$ & $15(39,5 \%)$ & \multirow[t]{4}{*}{$<0,001^{*}$} \\
\hline & II $(n=39)$ & $29(30,2 \%)$ & $10(26,3 \%)$ & \\
\hline & III $(n=10)$ & $4(4,2 \%)$ & $6(15,8 \%)$ & \\
\hline & IV $(n=58)$ & $51(53,1 \%)$ & $7(18,4 \%)$ & \\
\hline
\end{tabular}

Nota: *Associação linear.

\section{Discussão}

Nossos achados mostraram que o financiamento de publicações pela indústria, a respeito da terapia com PRP, está significativamente associado a resultados favoráveis. Além disso, observamos que quanto maior o nível de evidência, menor a proporção de resultados favoráveis. Ao mesmo tempo, reconhecemos as limitações da nossa análise, como não poder avaliar e detectar achados significativos em relação ao tipo de financiamento. Em muitos casos, não qualificamos o apoio em virtude de ele não ter sido descrito. No entanto, este artigo é o primeiro a descrever possíveis variáveis que podem influenciar os resultados de publicações sobre terapia com PRP.

O uso clínico e terapêutico da terapia com PRP tem sido estudado por muitos autores. ${ }^{7,12,13}$ Uma revisão sistemática ${ }^{7}$ que avaliou os efeitos (benéficos e maléficos) da terapia com PRP no tratamento das DOMs, no geral, concluiu que atualmente não existem evidências suficientes para apoiar o seu uso. ${ }^{7}$ Outra revisão sistemática ${ }^{12}$ avaliou os efeitos da terapia com PRP no tratamento de osteotomias de ossos longos, fraturas agudas, fraturas não unidas e defeitos, com os autores demonstrando que as evidências atualmente 
Tabela 4 Regressão logística binária das variáveis associadas a resultados favoráveis

\begin{tabular}{|c|c|c|c|c|}
\hline & & Coeficiente B & $\begin{array}{l}\text { Razão de probabilidade } \\
\text { ajustada (IC95\%) }\end{array}$ & Valor de $p$ \\
\hline \multirow[t]{3}{*}{ Financiamento } & Patrocinado pela indústria & - & - & 0,058 \\
\hline & Não patrocinado pela indústria & $-1,32$ & $0,26(0,08-0,85)$ & 0,026 \\
\hline & Não identificável & $-0,91$ & $0,39(0,12-1,30)$ & 0,130 \\
\hline \multirow[t]{4}{*}{ Nível de evidência } & I & - & - & $<0,001$ \\
\hline & II & 2,52 & $12,42(3,79-40,67)$ & $<0,001$ \\
\hline & III & 0,82 & $2,27(0,76-6,78)$ & 0,141 \\
\hline & IV & 2,39 & $10,97(2,33-51,51)$ & 0,002 \\
\hline
\end{tabular}

Abreviatura: IC95\%, intervalo de confiança de $95 \%$.

disponíveis são insuficientes para respaldar o uso rotineiro dessa intervenção na prática clínica. ${ }^{12}$ Uma metanálise ${ }^{13}$ estudou a eficácia do tratamento com PRP na lesão dos isquiotibiais e não mostrou efeito quando os pacientes foram comparados com o grupo controle (OR: 1,03; IC95\%: 0,87$1,22 ; p=0,73) .{ }^{13}$ Essas revisões parecem demonstrar que existem estudos com baixa qualidade de evidência, sendo que atualmente existem dados insuficientes demonstrando o benefício clínico com o uso da terapia com PRP.

Amiri et $\mathrm{l}^{14}$ encontraram resultados favoráveis na associação entre financiamento da pesquisa e as conclusões favoráveis em $81 \%$ das pesquisas sobre coluna vertebral. Em outro artigo, ${ }^{15}$ a taxa das pesquisas com resultados positivos foi de $48,8 \%$. No entanto, esses autores consideraram o resultado "neutro", com frequência de $41,4 \%$ no respectivo trabalho, e na artroplastia do quadril e do joelho, mostraram que 70,81\% dos artigos apresentaram resultados positivos. A maioria dos artigos apresentou viés favorável nos resultados. Da mesma forma, taxas baixas de conclusões desfavoráveis também foram relatadas por outros autores em pesquisas sobre ortopedia geral. ${ }^{16}$

Tem havido muito debate em torno do financiamento de pesquisa e o quanto esse patrocínio pode influenciar na publicação de estudos favoráveis. ${ }^{11,15,16}$ Nossos resultados mostraram que a indústria foi o patrocinador da pesquisa em $26,1 \%$ dos casos, mas, em quase metade das pesquisas, não foi possível classificar o financiador. Uma revisão de 886 artigos descreveu que 246 (27,7\%) projetos de pesquisa foram patrocinados pela indústria. ${ }^{11}$ Printz et al ${ }^{16}$ realizaram uma revisão crítica com 48 estudos sobre injeções de ácido hialurônico para osteoartrite do joelho, e descobriram que 35\% das publicações foram financiadas pelo setor industrial.

Noordin et al $^{15}$ constataram que os estudos patrocinados pela indústria tinham uma maior probabilidade de relatar resultados favoráveis em relação aos estudos patrocinados por outras fontes. ${ }^{15}$ Outros autores revisaram a relação do apoio financeiro com as conclusões positivas na pesquisa sobre a coluna vertebral, ${ }^{12}$ e mostraram que a RP era de 3,3 (IC95\%: 2,0-5,5). Em nossa pesquisa, observou-se que a RP ajustada foi de 0,26 (IC95\%: 0,08-0,85) nas publicações não patrocinadas pela indústria. Torna-se difícil comparar os resultados, considerando quantos casos do nosso ensaio foram classificados como "não identificáveis".
Nossos resultados também mostram os artigos sem a devida declaração de apoio financeiro à pesquisa. Outros autores corroboraram esses dados, com 41,3\% dos artigos sem informações relacionadas ao respaldo financeiro. ${ }^{17}$ Portanto, acreditamos que a frequência dos artigos patrocinados pelas indústrias do setor de saúde não pode ser determinada com exatidão por meio da metodologia utilizada. Ainda que a indústria, o profissional e o paciente tenham muitos interesses em comum, também pode haver interesses conflitantes reais ou potenciais. ${ }^{18}$ Um estudo ${ }^{19}$ analisou a divulgação de conflito de interesses entre ortopedistas em uma reunião da American Academy of Orthopaedic Surgeons, ${ }^{19}$ em que $20,7 \%$ dos profissionais não revelaram seus pagamentos diretamente relacionados. Dessa forma, observou-se que as políticas relativas a conflito de interesses e sua divulgação estão em constante fluxo, e que talvez seja necessário esclarecimentos adicionais às pessoas sobre as demandas de divulgação. ${ }^{11}$

O desafio é identificar e gerenciar o conflito, e, por conseguinte, vem a obrigação dos autores de divulgá-lo. ${ }^{20}$ Por outro lado, em nossa análise, corroborada por outros autores, muitos periódicos não exigem obrigatoriamente a declaração de conflitos de interesses. ${ }^{11}$

Nosso trabalho mostrou a presença de mais artigos com nível de evidência IV. Pinski et $\mathrm{al}^{21}$ avaliaram o nível de evidência nas pesquisas sobre o tratamento cirúrgico das lesões osteocondrais, e demonstraram que 90\% dos artigos tinham nível IV de evidência. Cunningham et $\mathrm{al}^{22}$ mostraram que as publicações dos níveis I e II, tanto quanto as dos níveis III e IV, aumentaram significativamente entre os anos de 2000 e 2010. Apesar disso, a proporção de estudos de nível IV sempre foi maior durante os 10 anos de análise.

Amiri et al ${ }^{12}$ mostraram que, entre os resultados positivos ( $80 \%$ dos casos), foram considerados $85 \%$ dos artigos de nível IV e $63 \%$ dos artigos de nível I. $^{12}$ Por outro lado, entre os resultados desfavoráveis, $14 \%$ dos estudos de nível I e $6 \%$ dos estudos de nível IV puderam ser observados. Esses achados mostraram uma relação linear significativa: quanto maior o nível de evidência, menor a frequência de estudos favoráveis. ${ }^{23}$

\section{Conclusão}

Portanto, nosso artigo mostrou que, em geral, resultados positivos são mais frequentes em publicações sobre terapia 
com PRP em DOMs. A maioria dos artigos não era afiliada academicamente, não declarou apoio financeiro à pesquisa, $\mathrm{e}$ tinha nível de evidência IV. Observamos que os estudos patrocinados pelo setor industrial tinham mais probabilidade de apresentar resultados positivos, além de artigos com menor qualidade de evidência. É crucial avaliar de forma crítica cada artigo científico, e não confiar cegamente nas conclusões de seus autores, além de estar ciente do possível conflito de interesses por parte dos autores que pesquisam essa área.

Conflito de Interesses

Os autores declaram não haver conflito de interesses.

\section{Agradecimentos}

Agradecemos ao nosso professor de epidemiologia e bioestatística, Petrônio Fagundes de Oliveira Filho, pela sua ajuda e seus ensinamentos. Somos gratos ao professor Robert Sweeney por toda a ajuda com a língua inglesa. Também gostaríamos de agradecer ao Instituto de Medicina do Esporte e Ciências Aplicadas ao Movimento Humano da Universidade de Caxias do Sul e a todos os seus colaboradores, especialmente aos amigos e médicos do esporte, Eduardo Pflug Comparsi e Olga Sergueevna Tairova.

\section{Referências}

1 Dohan Ehrenfest DM, Rasmusson L, Albrektsson T. Classification of platelet concentrates: from pure platelet-rich plasma (P-PRP) to leucocyte- and platelet-rich fibrin (L-PRF). Trends Biotechnol 2009;27(03):158-167

2 Foster TE, Puskas BL, Mandelbaum BR, Gerhardt MB, Rodeo SA. Platelet-rich plasma: from basic science to clinical applications. Am J Sports Med 2009;37(11):2259-2272

3 Ziltener JL, Didisheim C, Borloz S. Injections of Platelet-Rich Plasma (PRP) for the treatment of sports injuries: a review. Schweiz Z Med Traumatol 2012;60(04):157-160

4 Hamilton BH, Best TM. Platelet-enriched plasma and muscle strain injuries: challenges imposed by the burden of proof. Clin J Sport Med 2011;21(01):31-36

5 Buchkowsky SS, Jewesson PJ. Industry sponsorship and authorship of clinical trials over 20 years. Ann Pharmacother 2004;38 (04):579-585

6 Crowninshield R. The orthopaedic profession and industry: conflict or convergence of interests. Clin Orthop Relat Res 2003; (412):8-13

7 Moraes VY, Lenza M, Tamaoki MJ, Faloppa F, Belloti JC. Plateletrich therapies for musculoskeletal soft tissue injuries. Cochrane Database Syst Rev 2014;(04):CD010071
8 Khan SN, Mermer MJ, Myers E, Sandhu HS. The roles of funding source, clinical trial outcome, and quality of reporting in orthopedic surgery literature. Am J Orthop 2008;37(12): E205-E212

9 Leopold SS, Warme WJ, Fritz Braunlich E, Shott S. Association between funding source and study outcome in orthopaedic research. Clin Orthop Relat Res 2003;(415):293-301

10 Oxford centre for evidence-based medicine - level of evidence. 2009. Available at: http://www.cebm.net/index.aspx?051025

11 Fleiss JL, Levin B, Paik MC. Statistical methods for rates and proportions. $3^{\text {rd }}$ ed. Hoboken, New Jersey: John Wiley \& Sons; 2003

12 Griffin XL, Wallace D, Parsons N, Costa ML. Platelet rich therapies for long bone healing in adults. Cochrane Database Syst Rev 2012; (07):CD009496

13 Pas HI, Reurink G, Tol JL, Weir A, Winters M, Moen MH. Efficacy of rehabilitation (lengthening) exercises, platelet-rich plasma injections, and other conservative interventions in acute hamstring injuries: an updated systematic review and meta-analysis. Br J Sports Med 2015;49(18):1197-1205

14 Amiri AR, Kanesalingam K, Cro S, Casey AT. Does source of funding and conflict of interest influence the outcome and quality of spinal research? Spine J 2014;14(02):308-314

15 Noordin S, Wright JG, Howard A. Relationship between declared funding support and level of evidence. J Bone Joint Surg Am 2010; 92(07):1647-1651

16 Printz JO, Lee JJ, Knesek M, Urquhart AG. Conflict of interest in the assessment of hyaluronic acid injections for osteoarthritis of the knee: an updated systematic review. J Arthroplasty 2013;28(8, Suppl)30-33.e1

17 Bartels RH, Delye H, Boogaarts J. Financial disclosures of authors involved in spine research: an underestimated source of bias. Eur Spine J 2012;21(07):1229-1233

18 Singh N, Bush R, Dalsing M, Shortell CK. New paradigms for physician-industry relations: overview and application for SVS members. J Vasc Surg 2011;54(3, Suppl)26S-30S

19 Okike K, Kocher MS, Wei EX, Mehlman CT, Bhandari M. Accuracy of conflict-of-interest disclosures reported by physicians. N Engl J Med 2009;361(15):1466-1474

20 Bailey CS, Fehlings MG, Rampersaud YR, Hall H, Wai EK, Fisher CG. Industry and evidence-based medicine: Believable or conflicted? A systematic review of the surgical literature. Can J Surg 2011;54 (05):321-326

21 Pinski JM, Boakye LA, Murawski CD, Hannon CP, Ross KA, Kennedy JG. Low Level of Evidence and Methodologic Quality of Clinical Outcome Studies on Cartilage Repair of the Ankle. Arthroscopy 2016;32(01):214-22.e1

22 Cunningham BP, Harmsen S, Kweon C, Patterson J, Waldrop R, McLaren A, McLemore R. Have levels of evidence improved the quality of orthopaedic research? Clin Orthop Relat Res 2013;471 (11):3679-3686

23 Lynch JR, Cunningham MR, Warme WJ, Schaad DC, Wolf FM, Leopold SS. Commercially funded and United States-based research is more likely to be published; good-quality studies with negative outcomes are not. J Bone Joint Surg Am 2007;89(05):1010-1018 